\title{
New Recognition Elements for Bioanalytics
}

\author{
Frieder W.SCHELLER
}

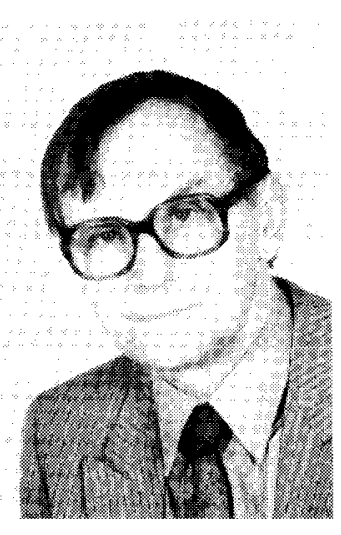

Enzymes and antibodies represent powerful tools that allow for sensitive and specific methods of detection and quantitation to be developed for a wide variety of substances. The basic principle behind the high chemical selectivity of these biomacromolecules is the structural complementarity of the recognition elements and the target analyte. In addition to the proteic macromolecules nucleic acid's and carbohydrates are increasingly used in specialized areas, for example for the sequencing of genomes and for cell surface characterization. The borderline between recognition tools that are of biological nature, and synthetic (organic) receptor molecules is no longer well definable; these two classes of recognition elements are merging. By mimicking the binding area of enzymes host molecules for binding the respective guests have been developed. Alternatively, molecular modeling is used to design appropriate binding structures which are combined as building blocks for signal generation, for example for sensing of glucose, ethanol and creatinine.

Molecular imprintings can be synthesized by forming a polymer network around a template. In this way structures complementary to the analyte are created on the surface of the polymeric carrier. Molecularly imprinted polymers have been applied for an increasing number of analytes in (bio) sensors, e.g. of D- and L-amino acids, atrazine, cholesterol, ephedrine, diazepam, and morphine.

Another synthetic approach uses oligonucleotides (aptamers) generated by random synthesis. In the next step the library is screened for the binding to the immobilized target molecule. The appropriate sequence is than amplified by a combination of the well established reverse transcription and polymerase chain reaction. Using this, highly innovative, approach a recognition partner has been synthesized that possesses a $10^{4}$ fold higher affinity constant for theophylline than for the very similar caffeine molecule. However, the application of aptamers in sensor arrangements has only just begun.

While the binding imprints and aptamers mimic the action of antibodies, the catalytic function of enzymes may be simulated by an analogous concept. By using (stable) analogues of the transition state as the template in the generation of antibodies, imprinted polymers and aptamers, in fact new catalytically active species have been generated. On the basis of this concept catalytic antibodies for the following reactions have been prepared : ester hydrolysis, amidohydrolysis, cyclization, amide bond formation, Claizen rearrangement, decarboxylation, peroxidation, cis-trans isomerization, $\beta$-elimination. $\mathrm{Up}$ to now, enzymes have been the better catalysts. with typical turn-over numbers of several ten thousands per second ; typical catalytic antibodies reach only one percent of this value.

The monograph "Frontiers in Biosensorics" by F.W. Scheller, F. Schubert and J. Fedrowitz (Birkhäuser Verlag, Basel, Switzerland) coming out at the end of this year presents comprehensively the fundamental aspects and practical applications of new recognition elements.

Professor, Analytical Biochemistry, Institute of Biochemistry and Molecular Physiology, University of Potsdam. (c./o. Max-Delbrück-Center of Molecular Medicine, Robert-Rössle-Str. 10, D-13122 Berlin, Germany) 